\title{
Polynomially solvable cases of the bipartite traveling salesman problem ${ }^{\text {光 }}$
}

\author{
Alfredo García ${ }^{a}$, Javier Tejel ${ }^{a, *}$ \\ ${ }^{a}$ Departamento de Métodos Estadísticos, IUMA, Universidad de Zaragoza, Spain.
}

\begin{abstract}
Given two sets, $R$ and $B$, consisting of $n$ cities each, in the bipartite traveling salesman problem one looks for the shortest way of visiting alternately the cities of $R$ and $B$, returning to the city of origin. This problem is known to be NP-hard for arbitrary sets $R$ and $B$. In this paper we provide an $O\left(n^{6}\right)$ algorithm to solve the bipartite traveling salesman problem if the quadrangle property holds. In particular, this algorithm can be applied to solve in $O\left(n^{6}\right)$ time the bipartite traveling salesman problem in the following cases: $S=R \cup B$ is a convex point set in the plane, $S=R \cup B$ is the set of vertices of a simple polygon and $V=R \cup B$ is the set of vertices of a circular graph. For this last case, we also describe another algorithm which runs in $O\left(n^{2}\right)$ time.
\end{abstract}

Keywords: Traveling salesman, Combinatorial optimization, Bipartite traveling salesman, quadrangle property, Kalmanson matrix, Transportation

\section{Introduction}

\subsection{Background and prior work}

In the traveling salesman problem (TSP), given a prescribed set of cities, one wishes to find the shortest route starting and finishing at the same location, visiting each one of the cities exactly once. This problem is perhaps one of the most extensively studied problems in combinatorial optimization and its different variants have many applications in different areas of knowledge, including computer science, operations research, genetics, engineering and electronics. The reader is referred to $[23,26]$ for a review of the state of the art on this problem.

One of these variants is the bipartite TSP (BTSP). Now, the set of cities is partitioned into two classes $R$ and $B$, with $|R|=|B|=n$, and one wishes to find a shortest route such that the cities in $R$ and $B$ alternate along the route. Besides being interesting in itself, the BTSP is related to other problems, mainly pickup and delivery problems, such as the

\footnotetext{
«Partially supported by Gobierno de Aragón under grant E58 (ESF) and projects MTM201230951(MINECO) and MTM2015-63791-R (MINECO/FEDER).

${ }^{*}$ Corresponding author

Email addresses: olaverri@unizar.es (Alfredo García), jtejel@unizar.es (Javier Tejel)
} 
pick-and-place robots problem (or the printed circuit board assembly problem) [29], the $k$-delivery TSP [1] or the swapping problem [4]. For an overview on pickup and delivery problems, see [8].

In the $k$-delivery TSP ( $k$-DTSP), one looks for the shortest route to pick up $n$ items located at $n$ source points and deliver them to $n$ sink points, using a single vehicle of capacity $k$ and assuming that an item at a source point can be delivered to any sink point. The BTSP is a special case of the $k$-DTSP if $k=1$. In the swapping problem, the main goal is to find the shortest route to swap $n$ objects of $m \leq n$ different types between $n$ locations using a single vehicle with unit capacity. Every location is associated with two object types - the one it currently holds and the one it demands- and holds or demands at most one unit of an object. Moreover, the total supply in the network for each object type equals its total demand. When there are only two object types, the swapping problem is equivalent to the BTSP. The reader is referred to $[1,9,11,31]$ and $[2,3,4,5]$ for different results and variants on the $k$-DTSP and the swapping problem, respectively.

In the Euclidean BTSP, the cities are assumed to be points in the plane and the distance between any two points is the Euclidean distance. It is well-known that the BTSP and the Euclidean BTSP are NP-hard, so there is no polynomial algorithm to solve them unless $P=N P$. Moreover, the problem remains NP-hard even in the case of a grid graph. In general, researchers have focused on designing good approximation algorithms. We refer the reader to $[4,7,12,20,28,29]$ and the references therein for different approximation algorithms along with experimental results. The best known approximation factor for the Euclidean BTSP is $2[12,20]$.

There are also some publications in the literature devoted to solving particular cases of the BTSP. In [31], the authors study the $k$-DTSP for path and tree graphs. In the case of a path, they give an $O\left(n^{2} / \min \{k, n\}\right)$ algorithm for arbitrary $k$ and linear algorithms for $k=1$ and $k=\infty$. In the case of a tree (see also [3]), they propose an $O\left(n^{2}\right)$ algorithm for $k=1$ and an $O(n)$ algorithm for $k=\infty$, and show that the problem becomes NP-hard in strong sense if $k$ is arbitrary. In [9], a linear-time algorithm is described to solve the $k$-DTSP on a path.

Another particular case of the BTSP studied in the literature is related to the shoelace problem [24]. In this problem, the objective is to find an optimal strategy for lacing shoes such that the amount of shoelace used is minimized. When the eyelets are arranged in two horizontal lines and two eyelets on the same line are not connected consecutively, then the shoelace problem is an instance of the BTSP (the eyelets placed on the two lines correspond to $R$ and $B$, respectively). In this case, Halton [24] proved that the optimal way of threading the shoelaces is the so-called criss-cross lacing strategy, which corresponds to the typical method used in the USA for lacing shoes: threading the shoelaces in opposing zigzags, so that they seem to be crossed when seen from above.

Halton's result was generalized later in [27] and [19]. In both papers the authors show that the criss-cross strategy is still the best way of visiting the cities under certain constraints on the distance matrix $D$. If $1,2, \ldots, n$ and $n+1, n+2, \ldots, 2 n$ are the cities in $R$ and $B$, respectively, Misiurewicz [27] shows that it is sufficient for the distance matrix $D$ to satisfy: $d(i, j)+d(k, l) \leq d(i, l)+d(k, j)$ for $1 \leq i \leq k \leq n$ and $n+1 \leq j \leq l \leq 2 n$. 

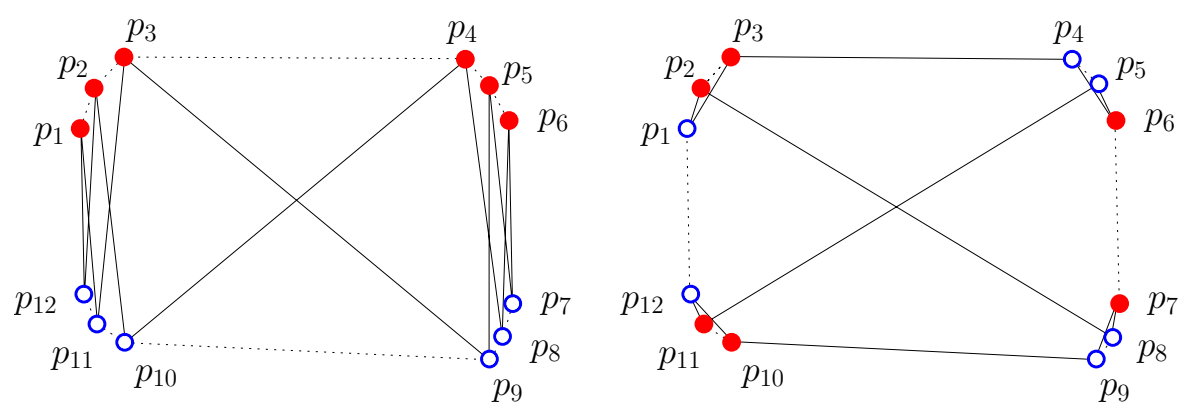

Figure 1: Left: The shortest Hamiltonian alternating cycle is the criss-cross cycle. Right: The shortest Hamiltonian alternating cycle for another partition of the same convex point set.

In the second paper [19], Deineko and Woeginger prove the result for a relaxation on the Monge inequalities for a matrix $M$ and provide an $O\left(n^{4}\right)$ algorithm to decide whether there is a renumbering of the cities such that the resulting distance matrix satisfies this relaxation.

\subsection{The quadrangle property}

A classic example where Misiurewicz's conditions are satisfied is the following. Consider the set of $2 n$ vertices of a convex polygon and suppose that the clockwise order of the vertices is $\{1,2, \ldots, 2 n\}$. Assume that the vertices from 1 to $n$ belong to $R$, the vertices from $n+1$ to $2 n$ belong to $B$, and that the cost of an edge connecting one vertex to another is the Euclidean distance. The well-known quadrangle property for a convex quadrilateral states that the total length of the diagonals of the quadrilateral is always bigger than the total length of two opposite sides. In particular, given any four vertices $i<k<j<l$, with $i$ and $k$ belonging to $R$ and $j$ and $l$ to $B$, the total length of the two crossing edges $(i, j)$ and $(k, l)$ is always bigger than the total length of the two non-crossing edges $(i, l)$ and $(k, j)$. These are Misiurewicz's conditions for reversing the order of the vertices of $B$. Therefore, the shortest way of visiting alternately the vertices in $R$ and $B$ is the criss-cross cycle, as is shown in the left part of Figure 1. Vertices belonging to $R$ are illustrated as solid red points and vertices belonging to $B$ as hollow blue points.

Assume now that the $2 n$ vertices of the convex polygon are divided into two arbitrary sets $R$ and $B$ of equal size, as in the right part of Figure 1. Misiurewicz's inequalities are no longer satisfied because the vertices in $R$ and $B$ are not consecutive in the cyclic order. However, it is still true that if two edges (segments) of the bipartite graph defined by $R$ and $B$ cross, then they can be replaced by two other edges (segments) of the bipartite graph, reducing the total length. Using this fact, one can still compute the shortest Hamiltonian cycle $C$ visiting alternately the vertices in $R$ and $B$, as the right part of Figure 1 shows.

These are precisely the types of particular cases of the BTSP we study in this paper: instances in which, given a cyclic order on the cities, two "crossing edges" can be replaced by two "non-crossing edges" without increasing the length. This concept is formalized for graphs as follows.

Definition 1. Let $G=(V, E)$ be an undirected graph on the set of vertices $V=\{1,2, \ldots, N\}$. 
For an edge $e=(i, j)$ of $E$, let $d(i, j)$ be the cost of $e$. Assuming that $(1,2, \ldots, N)$ is a cyclic order of the vertices of $G$, we say that $G$ satisfies the quadrangle property if

$$
d\left(i_{1}, i_{4}\right)+d\left(i_{2}, i_{3}\right) \leq d\left(i_{1}, i_{3}\right)+d\left(i_{2}, i_{4}\right)
$$

for any four vertices $i_{1}, i_{2}, i_{3}, i_{4}$ such that $\left(i_{1}, i_{4}\right),\left(i_{2}, i_{3}\right),\left(i_{1}, i_{3}\right),\left(i_{2}, i_{4}\right)$ are edges of $E$ and $i_{1}<i_{2}<i_{3}<i_{4}$ cyclically.

These inequalities, $d\left(i_{1}, i_{4}\right)+d\left(i_{2}, i_{3}\right) \leq d\left(i_{1}, i_{3}\right)+d\left(i_{2}, i_{4}\right)$, are usually called quadrangle inequalities. Misiurewicz's conditions correspond to the quadrangle property for the particular case of the complete bipartite graph $G=(R \cup B, E)$, with $R=\{1,2, \ldots, n\}$, $B=\{n+1, n+2, \ldots, 2 n\}$ and the cyclic order $(1,2, \ldots n, 2 n, 2 n-1, \ldots, n+1)$.

\subsection{Our main contribution}

In this paper, we study the BTSP for a complete bipartite graph $G=(R \cup B, E)$ satisfying the quadrangle property, that is, assuming that $(1,2, \ldots, 2 n)$ is a cyclic order of the vertices of $G$, inequality $d\left(i_{1}, i_{4}\right)+d\left(i_{2}, i_{3}\right) \leq d\left(i_{1}, i_{3}\right)+d\left(i_{2}, i_{4}\right)$ holds for any four vertices $i_{1}, i_{2} \in R$, and $i_{3}, i_{4} \in B$ such that $i_{1}<i_{2}<i_{3}<i_{4}$ cyclically. To the best of our knowledge, this problem has only been solved when $R=\{1,2, \ldots, n\}$ and $B=\{n+1, n+2, \ldots, 2 n\}$ (Misiurewicz [27]). We show that there is a shortest cycle for the BTSP in $G$ not containing a five-point star (defined later). Then, we provide an $O\left(n^{6}\right)$ algorithm to find the shortest cycle not containing a five-point star. As a consequence, we also solve in $O\left(n^{6}\right)$ time the following three particular cases of the BTSP: the Euclidean BTSP for points in convex position, the BTSP for a simple polygon and the BTSP for a circular graph (or cycle graph). In the three cases, the quadrangle property holds. Line and circular graph models have been widely studied in the literature, because of their real applications: Trucks delivering goods along a highway or ships visiting ports along a shoreline are some examples. Simple polygons are often used to model terrains and can also be used, for example, to model a lake in which boats have to visit ports placed on the shoreline, following the shortest paths among the ports.

\subsection{Organization of the paper}

The paper is organized as follows. Section 2 is devoted to studying the BTSP for a complete bipartite graph satisfying the quadrangle property with a given cyclic order on its vertices. In particular, we show that there is a shortest cycle not containing a five-point star. In Section 3, we describe an $O\left(n^{6}\right)$ algorithm to find the shortest cycle not containing a five-point star, improving the best known algorithm for this problem that runs in $O\left(n^{7}\right)$ time [18]. In Section 4, we show three particular cases satisfying the quadrangle property: the BTSP for convex point sets, for simple polygons and for circular graphs. We also analyze the case of the BTSP for Kalmanson matrices. In Section 5, we analyze in more detail the structure of cycles not containing five-point stars and we give an $O\left(n^{2}\right)$ algorithm to solve the BTSP for a circular graph. Hence, we also solve in $O\left(n^{2}\right)$ time the swapping problem for circular graphs with $m=2$ object types. The complexity of the general swapping problem for a circular graph is unknown $[3,5]$. Finally, some concluding remarks are given in Section 6. 


\section{The BTSP for a complete bipartite graph satisfying the quadrangle property}

Before studying the BTSP for a complete bipartite graph, we give some notation and definitions which are used throughout the paper. $G=(V, E)$ is an undirected graph on the set of vertices $V=\{1,2, \ldots, N\}$, where $(1,2, \ldots, N)$ is assumed to be a cyclic order of the vertices of $V$. For an edge $e=(i, j)$ of $G, d(i, j)$ is the cost associated with $e$ (or the length of $e$ or the distance between vertices $i$ and $j$ ).

A Hamiltonian cycle $C$ on $G$ is given by a cyclic permutation $\left(i_{1}, i_{2}, \ldots, i_{N}\right)$ of the vertices of $G$, so $C$ consists of the edges $\left(i_{1}, i_{2}\right),\left(i_{2}, i_{3}\right) \ldots,\left(i_{N}, i_{1}\right)$ of $G$. We consider $C$ as a directed cycle consisting of the directed edges $\left(i_{1}, i_{2}\right),\left(i_{2}, i_{3}\right) \ldots,\left(i_{N}, i_{1}\right)$. The length of $C$ is the amount

$$
l(C)=\sum_{j=1}^{N} d\left(i_{j}, i_{j+1}\right)
$$

where $i_{N+1}$ is identified with $i_{1}$.

We say that two edges $\left(i_{1}, i_{3}\right)$ and $\left(i_{2}, i_{4}\right)$ of $G$ combinatorially cross if and only if the cyclic order of the four vertices is either $i_{1}<i_{2}<i_{3}<i_{4}$ or $i_{1}<i_{4}<i_{3}<i_{2}$. We also say that two edges which cross define a combinatorial crossing. Using the following trivial observation, one can imagine combinatorial crossings as geometric crossings between segments connecting points in the plane in convex position.

Observation 1. Let $\left\{p_{1}, p_{2}, \ldots, p_{N}\right\}$ be a set of $N$ points in the plane on a unit circle, clockwise ordered. Let $G=(V, E)$ be a graph on the set of vertices $V=\{1,2, \ldots, N\}$, where $(1,2, \ldots, N)$ is assumed to be a cyclic order of the vertices of $V$. Identifying vertex $k$ with point $p_{k}$, for $k=1, \ldots, N$, two edges $(i, j)$ and $\left(i^{\prime}, j^{\prime}\right)$ of $G$ combinatorially cross if and only if their corresponding segments $p_{i} p_{j}$ and $p_{i^{\prime}} p_{j^{\prime}}$ geometrically cross.

Optimum cycles are defined in the following way.

Definition 2. A Hamiltonian cycle $C$ in $G$ is optimum if its length is less than or equal to the length of any other Hamiltonian cycle $C^{\prime}$ and, if both cycles have the same length, the number of combinatorial crossings of $C$ is less than or equal to the number of combinatorial crossings of $C^{\prime}$.

Given a partition $R \cup B$ of the set of vertices of $G$ into two sets $R$ and $B$, we say that $R$ is the set of red vertices and $B$ is the set of blue vertices. A cycle (path) is alternating if any two consecutive vertices have distinct color. Lastly, a five-point star for a cyclic permutation is defined as follows.

Definition 3. Let $\pi=\left(i_{1}, i_{2}, \ldots, i_{N}\right)$ be a cyclic permutation of the set $\{1,2, \ldots, N\}$. Given five arbitrary indices $\left\{j_{1}, j_{2}, j_{3}, j_{4}, j_{5}\right\}$ such that $j_{1}<j_{2}<j_{3}<j_{4}<j_{5}$ cyclically, we say that the five indices form a five-point star if they appear in $\pi$ in the order $\ldots, j_{1}, \ldots, j_{3}, \ldots, j_{5}, \ldots, j_{2}, \ldots, j_{4}, \ldots$ or in the order $\ldots, j_{1}, \ldots, j_{4}, \ldots, j_{2}, \ldots, j_{5}, \ldots, j_{3}, \ldots$.

Let $G=(R \cup B, E)$ be a complete bipartite graph such that $R \cup B$ is a partition of $V=\{1,2, \ldots, 2 n\}$, with $|R|=|B|=n$. In the BTSP for $G$, we look for a Hamiltonian 
alternating cycle $C$ such that $l(C) \leq l\left(C^{\prime}\right)$ for any other Hamiltonian alternating cycle $C^{\prime}$. Assuming that $G$ satisfies the quadrangle property with respect to the cyclic order $(1,2, \ldots, 2 n)$, the main result of this section is that there is a shortest Hamiltonian alternating cycle not containing a five-point star. To prove this result, we need two lemmas. The first lemma shows that, if two crossing edges of a subgraph of $G$ are replaced by two non-crossing edges of $G$, then the number of combinatorial crossings decreases. The second lemma gives a constraint on the colors of the endpoints of crossing edges in an optimum Hamiltonian alternating cycle.

The first lemma is essentially the following well-known property for geometric graphs drawn on $n$ points in the plane in convex position, assuming that no three of them are on a line and that the distance between two points is the Euclidean distance. Recall that a geometric graph is a graph whose vertices are points in the plane and whose edges are straight line segments connecting the vertices.

Property 1. If $H$ is a geometric graph drawn on a convex point set $S$ such that edge $\left(p_{i}, p_{j}\right)$ crosses edge $\left(p_{i^{\prime}}, p_{j^{\prime}}\right)$, then the geometric graph $H^{\prime}$ obtained by replacing those edges by edges $\left(p_{i}, p_{i^{\prime}}\right)$ and $\left(p_{j}, p_{j^{\prime}}\right)$ (or by $\left(p_{i}, p_{j^{\prime}}\right)$ and $\left(p_{j}, p_{i^{\prime}}\right)$ ) has less total length than $H$. Moreover, $H^{\prime}$ also has fewer crossings than $H$.

This property follows from the convexity of $S$. For four points in convex position, with no three of them on a line, the quadrangle inequality is strict when using the Euclidean distance, so $H^{\prime}$ must have less length than $H$. On the other hand, if a crossing in $H^{\prime}$ does not involve edges $\left(p_{i}, p_{i^{\prime}}\right)$ and $\left(p_{j}, p_{j^{\prime}}\right)$, then this crossing also appears in $H$. Moreover, if an edge $e$ of $H^{\prime}$ crosses $\left(p_{i}, p_{i^{\prime}}\right)$ (or $\left(p_{j}, p_{j^{\prime}}\right)$ ), then, by convexity, $e$ necessarily crosses at least one of $\left(p_{i}, p_{j}\right)$ and $\left(p_{i^{\prime}}, p_{j^{\prime}}\right)$, and if $e$ crosses $\left(p_{i}, p_{i^{\prime}}\right)$ and $\left(p_{j}, p_{j^{\prime}}\right)$, then $e$ also crosses $\left(p_{i}, p_{j}\right)$ and $\left(p_{i^{\prime}}, p_{j^{\prime}}\right)$. Therefore, the number of crossings in $H$ is at least the number of crossings in $H^{\prime}$. Since $\left(p_{i}, p_{i^{\prime}}\right)$ and $\left(p_{j}, p_{j^{\prime}}\right)$ do not cross, the number of crossings in $H^{\prime}$ is fewer than the number of crossings in $H$.

From the definition of the quadrangle property, Observation 1 and Property 1, the following lemma holds.

Lemma 1. Let $G=(R \cup B, E)$ be a complete bipartite graph such that $R \cup B$ is a partition of $V=\{1,2, \ldots, 2 n\}$, with $|R|=|B|=n$. Assume that $G$ satisfies the quadrangle property with respect to the cyclic order $(1,2, \ldots, 2 n)$. If $H$ is a subgraph of $G$ such that edge $(i, j)$ combinatorially crosses edge $\left(i^{\prime}, j^{\prime}\right)$, then, by replacing those edges by edges $\left(i, i^{\prime}\right)$ and $\left(j, j^{\prime}\right)$ (if $i$ and $i^{\prime}$ have different colors) or by $\left(i, j^{\prime}\right)$ and $\left(j, i^{\prime}\right)$ (if $i$ and $i^{\prime}$ have the same color), the length of the resulting graph $H^{\prime}$ is less than or equal to the length of $H$. Moreover, $H^{\prime}$ has fewer combinatorial crossings than $H$.

Proof. Let $S=\left\{p_{1}, p_{2}, \ldots, p_{2 n}\right\}$ be a set of $2 n$ points in the plane on a unit circle, clockwise ordered. Let $\bar{H}=(S, \bar{E})$ and $\bar{H}^{\prime}=\left(S, \bar{E}^{\prime}\right)$ be the geometric graphs on $S$ associated with $H$ and $H^{\prime}$, respectively, where an edge (segment) $\left(p_{i}, p_{j}\right)$ belongs to $\bar{H}\left(\bar{H}^{\prime}\right)$ if and only if the edge $(i, j)$ belongs to $H\left(H^{\prime}\right)$. By Observation 1 , the number of combinatorial crossings of $H\left(H^{\prime}\right)$ must coincide with the number of geometric crossings of $\bar{H}\left(\bar{H}^{\prime}\right)$. Since $\bar{H}^{\prime}$ has been 

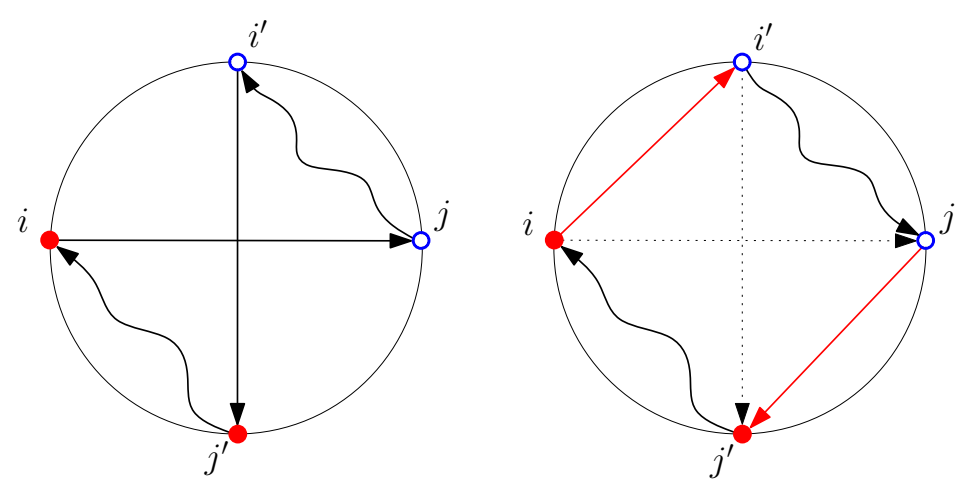

Figure 2: Replacing edges $(i, j)$ and $\left(i^{\prime}, j^{\prime}\right)$ by edges $\left(i, i^{\prime}\right)$ and $\left(j, j^{\prime}\right)$, a shorter Hamiltonian alternating cycle is obtained.

obtained from $\bar{H}$ by replacing two crossing edges by two non-crossing edges, by Property $1, \bar{H}^{\prime}$ has fewer geometric crossings than $\bar{H}$, so $H^{\prime}$ has fewer combinatorial crossings than $H$.

On the other hand, since edges $(i, j)$ and $\left(i^{\prime}, j^{\prime}\right)$ combinatorially cross, the cyclic order of the vertices is either $i^{\prime}<j<j^{\prime}<i$ or $i^{\prime}<i<j^{\prime}<j$. In both cases, as $G$ satisfies the quadrangle property, the sum of the lengths of the edges $\left(i, i^{\prime}\right)$ and $\left(j, j^{\prime}\right)$ (or the edges $\left(i, j^{\prime}\right)$ and $\left.\left(j, i^{\prime}\right)\right)$ is not bigger than the sum of the lengths of the edges $(i, j)$ and $\left(i^{\prime}, j^{\prime}\right)$. Hence, the length of $H^{\prime}$ is less than or equal to the length of $H$.

Lemma 2. Let $G=(R \cup B, E)$ be a complete bipartite graph such that $R \cup B$ is a partition of $V=\{1,2, \ldots, 2 n\}$, with $|R|=|B|=n$. Assume that $G$ satisfies the quadrangle property with respect to the cyclic order $(1,2, \ldots, 2 n)$. Let $C$ be an optimum Hamiltonian alternating cycle in $G$. If the directed edges $(i, j)$ and $\left(i^{\prime}, j^{\prime}\right)$ of $C$ combinatorially cross, then $i, i^{\prime}$ have the same color (and $j, j^{\prime}$ the other color).

Proof. Suppose that vertices $i$ and $i^{\prime}$ have different colors. Then, by replacing the crossing directed edges $(i, j)$ and $\left(i^{\prime}, j^{\prime}\right)$ by the directed edges $\left(i, i^{\prime}\right)$ and $\left(j, j^{\prime}\right)$ and by reversing the path of $C$ from $j$ to $i^{\prime}$, we obtain a new Hamiltonian alternating cycle $C^{\prime}$. Figure 2 illustrates the new cycle obtained, where paths connecting vertices are drawn as curves. By Lemma 1, $C^{\prime}$ has fewer crossings than $C$ and its length is at most the length of $C$, contradicting that $C$ is optimum.

Using the two previous lemmas, we now show that no optimum Hamiltonian alternating cycle can contain a five-point star.

Theorem 1. Let $G=(R \cup B, E)$ be a complete bipartite graph such that $R \cup B$ is a partition of $V=\{1,2, \ldots, 2 n\}$, with $|R|=|B|=n$. Assume that $G$ satisfies the quadrangle property with respect to the cyclic order $(1,2, \ldots, 2 n)$. Then, no optimum Hamiltonian alternating cycle $C$ in $G$ can contain a five-point star.

Proof. The proof is by contradiction. We show that if five vertices $i_{1}<i_{2}<i_{3}<i_{4}<i_{5}$ appear in $C$ in the cyclic order $i_{1}, \ldots, i_{3}, \ldots, i_{5}, \ldots, i_{2}, \ldots, i_{4}, \ldots$, then there are two pairs 

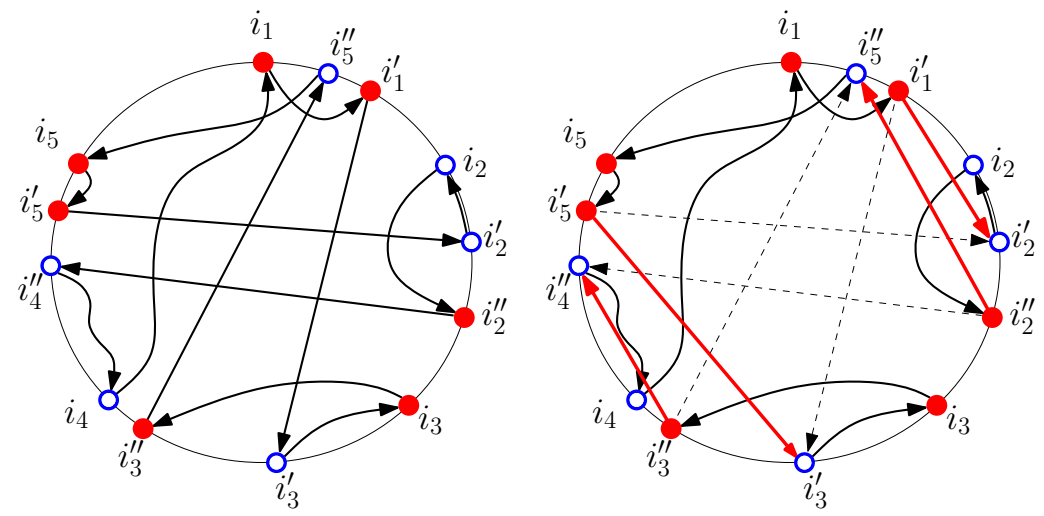

Figure 3: Building cycle $C^{\prime}$ (right) from cycle $C$ (left). The directed edges edges $\left(i_{5}^{\prime}, i_{2}^{\prime}\right),\left(i_{1}^{\prime}, i_{3}^{\prime}\right),\left(i_{2}^{\prime \prime}, i_{4}^{\prime \prime}\right)$ and $\left(i_{3}^{\prime \prime}, i_{5}^{\prime \prime}\right)$ of $C$ are replaced by the directed edges $\left(i_{5}^{\prime}, i_{3}^{\prime}\right),\left(i_{1}^{\prime}, i_{2}^{\prime}\right),\left(i_{2}^{\prime \prime}, i_{5}^{\prime \prime}\right)$ and $\left(i_{3}^{\prime \prime}, i_{4}^{\prime \prime}\right)$.

of crossing edges in $C$ such that, when replacing the crossing edges by the corresponding non-crossing edges, a new cycle shorter than $C$ is obtained. The same reasoning can be applied to the symmetric order $i_{1}, \ldots, i_{4}, \ldots, i_{2}, \ldots, i_{5}, \ldots, i_{3}, \ldots$

As $i_{1}<i_{2}<i_{3}<i_{5}$ cyclically, in the directed path of $C$ from $i_{5}$ to $i_{2}$, there is necessarily a directed edge $\left(i_{5}^{\prime}, i_{2}^{\prime}\right)$ such that the cyclic order of $i_{1}, i_{2}^{\prime}, i_{3}$ and $i_{5}^{\prime}$ is $i_{1}<i_{2}^{\prime}<i_{3}<i_{5}^{\prime}$. For the same reason, since $i_{1}<i_{2}^{\prime}<i_{3}<i_{5}^{\prime}$ cyclically, in the directed path of $C$ from $i_{1}$ to $i_{3}$ there is a directed edge $\left(i_{1}^{\prime}, i_{3}^{\prime}\right)$ such that $i_{1}^{\prime}<i_{2}^{\prime}<i_{3}^{\prime}<i_{5}^{\prime}$. These two directed edges $\left(i_{5}^{\prime}, i_{2}^{\prime}\right)$ and $\left(i_{1}^{\prime}, i_{3}^{\prime}\right)$ combinatorially cross, so $i_{5}^{\prime}$ and $i_{1}^{\prime}$ must have the same color by Lemma 2 and $i_{2}^{\prime}$ and $i_{3}^{\prime}$ the other color.

Using the same argument, in the paths of $C$ from $i_{2}$ to $i_{4}$ and from $i_{3}$ to $i_{5}$, there are two directed edges, $\left(i_{2}^{\prime \prime}, i_{4}^{\prime \prime}\right)$ in the path from $i_{2}$ to $i_{4}$ and $\left(i_{3}^{\prime \prime}, i_{5}^{\prime \prime}\right)$ in the path from $i_{3}$ to $i_{5}$ such that $i_{2}^{\prime \prime}<i_{3}^{\prime \prime}<i_{4}^{\prime \prime}<i_{5}^{\prime \prime}$. By Lemma $2, i_{2}^{\prime \prime}$ and $i_{3}^{\prime \prime}$ have the same color and $i_{4}^{\prime \prime}$ and $i_{5}^{\prime \prime}$ the other color.

Therefore, $C$ is $\left(i_{1}^{\prime}, i_{3}^{\prime}\right), P_{i_{3}^{\prime}, i_{3}^{\prime \prime}},\left(i_{3}^{\prime \prime}, i_{5}^{\prime \prime}\right), P_{i_{5}^{\prime \prime}, i_{5}^{\prime}},\left(i_{5}^{\prime}, i_{2}^{\prime}\right), P_{i_{2}^{\prime}, i_{2}^{\prime \prime}},\left(i_{2}^{\prime \prime}, i_{4}^{\prime \prime}\right), P_{i_{4}^{\prime \prime}, i_{1}^{\prime}}$, where $P_{i, j}$ is the directed path of $C$ from vertex $i$ to vertex $j$. Note that some of these paths $P_{i, j}$ can be empty. This happens when, for example, $i_{3}=i_{3}^{\prime}=i_{3}^{\prime \prime}$. Now, replacing the crossing edges $\left(i_{5}^{\prime}, i_{2}^{\prime}\right)$ and $\left(i_{1}^{\prime}, i_{3}^{\prime}\right)$ by the edges $\left(i_{5}^{\prime}, i_{3}^{\prime}\right)$ and $\left(i_{1}^{\prime}, i_{2}^{\prime}\right)$ and the crossing edges $\left(i_{2}^{\prime \prime}, i_{4}^{\prime \prime}\right)$ and $\left(i_{3}^{\prime \prime}, i_{5}^{\prime \prime}\right)$ by the edges $\left(i_{2}^{\prime \prime}, i_{5}^{\prime \prime}\right)$ and $\left(i_{3}^{\prime \prime}, i_{4}^{\prime \prime}\right)$, we obtain a new cycle $C^{\prime}=\left(i_{1}^{\prime}, i_{2}^{\prime}\right), P_{i_{2}^{\prime}, i_{2}^{\prime \prime}},\left(i_{2}^{\prime \prime}, i_{5}^{\prime \prime}\right), P_{i_{5}^{\prime \prime}, i_{5}^{\prime}}$, $\left(i_{5}^{\prime}, i_{3}^{\prime}\right), P_{i_{3}^{\prime}, i_{3}^{\prime \prime}},\left(i_{3}^{\prime \prime}, i_{4}^{\prime \prime}\right), P_{i_{4}^{\prime \prime}, i_{1}^{\prime}}$. Figure 3 illustrates how to obtain this cycle, assuming that the vertices of $G$ are points on a unit circle. By Lemma $1, C^{\prime}$ is not longer than $C$ and has fewer crossings than $C$, contradicting that $C$ is optimum.

\section{The five-point star algorithm}

In this section, we describe an $O\left(n^{6}\right)$ algorithm to compute the shortest cycle not containing a five-point star. This algorithm is the base for solving the particular cases of the BTSP studied in this paper.

Recall that, given a cyclic permutation $\pi=\left(i_{1}, i_{2}, \ldots, i_{n}\right)$ of the set $\{1,2, \ldots, n\}$, five indices $j_{1}<j_{2}<j_{3}<j_{4}<j_{5}$ form a five-point star if they appear in $\pi$ in the order 
$\left(\ldots, j_{1}, \ldots, j_{3}, \ldots, j_{5}, \ldots, j_{2}, \ldots, j_{4}, \ldots\right)$ or in the order $\left(\ldots, j_{1}, \ldots, j_{4}, \ldots, j_{2}, \ldots, j_{5}, \ldots, j_{3}, \ldots\right)$. Given a set of points $S$ in the plane, this class of permutations was defined in [21] as part of the characterization of the order in which the points on the second convex hull of $S$ are visited in any simple polygon. In the same paper, the authors give some alternative definitions for these permutations: g-pyramidal permutations and permutations satisfying the subdivision property. A permutation $\pi$ of $\{1,2, \ldots, n\}$ satisfies the subdivision property if it is formed by, first, a permutation $\pi_{1}$ of consecutive indices and later a permutation $\pi_{2}$ of the remaining indices and, besides, this division process can be repeated again for $\pi_{1}$ and $\pi_{2}$. A permutation is g-pyramidal, if it belongs to the family $\mathcal{G}_{n}$, defined recursively as follows:

(i) $\mathcal{G}_{1}=(1)$

(ii) The permutation $\pi_{n}$ belongs to $\mathcal{G}_{n}$ if and only if two adjacent and consecutive indices exist in $\pi_{n}$, and, by contracting them, the new permutation $\pi_{n-1}$ belongs to $\mathcal{G}_{n-1}$.

The following lemma, proved in [21], shows that these three definitions are equivalent.

Lemma 3. [21] A permutation is g-pyramidal $\Leftrightarrow$ it satisfies the subdivision property $\Leftrightarrow$ it does not contain a five-point star.

For example, the permutation (124386759) is g-pyramidal because we are able to make the following contractions: $(\mathbf{1 2 4 3 8 6 7 5 9 )} \rightarrow(13275648) \rightarrow(1326547) \rightarrow(132546) \rightarrow$ $(13245) \rightarrow(1324) \rightarrow(123) \rightarrow(12) \rightarrow(1)$. Moreover, it is clear that $(124386759)$ does not contain a five-point star and that it satisfies the subdivision property: (124386759) $\rightarrow$ $((1243)(86759)) \rightarrow(((12)(43))((8675)(9))) \rightarrow \ldots$

G-pyramidal permutations are generalizations of pyramidal permutations. A permutation $\pi=\left(1, i_{1}, i_{2}, \ldots, i_{r}, n, j_{1}, j_{2}, \ldots, j_{n-r-2}\right)$ of the set $\{1,2, \ldots, n\}$ is pyramidal if $i_{1}<$ $i_{2}<\ldots<i_{r}$ and $j_{1}>j_{2}>\ldots>j_{n-r-2}$. In a pyramidal permutation, indices $n$ and $n-1$ always appear consecutively in the permutation and, if these two indices are contracted, the resulting permutation is again pyramidal. Hence, the set of pyramidal permutations is a subset of the set of g-pyramidal permutations. In addition, while the number of pyramidal permutations is $2^{n-1}$, the number of g-pyramidal permutations is asymptotically $(3+\sqrt{8})^{n}$ $[15,30]$. The relevance of pyramidal permutations relies on the fact that, although the number of pyramidal permutations is exponential, finding an optimal pyramidal permutation (given a distance matrix) only requires $O\left(n^{2}\right)$ time. Thus, if one can prove that the optimal solution for a problem belongs to this family, then the problem can be solved polynomially. This happens, for example, when solving the TSP with a Demidenko distance matrix: One can prove that there is an optimal cycle belonging to the family of pyramidal permutations. The reader interested in other well-solvable cases of the TSP is referred to [10], chapter 11 in [23] and chapter 4 in [26].

G-pyramidal permutations are also known as twisted sequences. Twisted sequences were described by Aurenhammer in [6] and can be defined as follows. Start with the identity permutation $(1,2, \ldots, n)$ and choose a set of intervals over the set $\{1,2, \ldots, n\}$ such that for every pair of intervals either one of them contains the other one or the two intervals 
are disjoint. Then, reverse (twist) for every interval the order of its elements. A twisted sequence is obtained from the identity via such a reversal process $[6,18]$.

Given a distance matrix, in [18], Deineko et al. developed an $O\left(n^{7}\right)$ algorithm to compute the shortest twisted sequence. Their algorithm is based on proving that an optimal path that visits the indices $\{i, \ldots, j\}$ in a twisted way, starting and finishing at two indices $a, b \in[i, j]$, can be decomposed into two optimal paths visiting the indices in $[i, k]$ and $[k+1, j]$ in a twisted way, respectively. Although they did not give an explicit name to this property, it corresponds to the subdivision property. Hence, a twisted sequence satisfies the subdivision property. Besides, it is not difficult to see that a permutation satisfying the subdivision property is also a twisted sequence and then, the family of twisted sequences is the same as the family of g-pyramidal permutations.

Using the subdivision property, we can design a dynamic programming algorithm to find the shortest g-pyramidal cycle (or shortest twisted sequence). Given an $n \times n$ distance matrix $D$, where $d(i, j)$ is the distance between index $i$ and index $j$, the length of a cycle $C=\left(i_{1}, i_{2}, \ldots, i_{n}\right)$ is $l(C)=\sum_{j=1}^{n-1} d\left(i_{j}, i_{j+1}\right)+d\left(i_{n}, i_{1}\right)$. Given the distance matrix $D$, the output of the algorithm is the shortest g-pyramidal cycle $C$, that is, the g-pyramidal cycle $C$ such that $l(C) \leq l\left(C^{\prime}\right)$ for any other g-pyramidal cycle $C^{\prime}$.

The algorithm works as follows. Let us denote by $[i, j]$ the interval of consecutive indices $i, i+1, \ldots, j$ (assuming that $n$ is followed by 1 ). For each interval $[i, j]$, index $s \in[i, j]$ and index $t \notin[i, j]$, let $C(i, j, s, t)$ be the length of an optimal path beginning at $s$, visiting all the indices of $[i, j]$ and finishing at $t$, with the additional condition that the path visiting indices $[i, j]$ satisfies the subdivision property.

Clearly, if $[i, j]$ only contains one index $i$, then $C(i, i, i, t)=d(i, t)$. In any other case, by the optimality principle of dynamic programming, $C(i, j, s, t)$ is the minimum of:

$$
\left\{\begin{array}{l}
C\left(i, k, s, t^{\prime}\right)+C\left(k+1, j, t^{\prime}, t\right), k \in[s, j-1], t^{\prime} \in[k+1, j] \\
C\left(k+1, j, s, t^{\prime}\right)+C\left(i, k, t^{\prime}, t\right), k \in[i, s-1], t^{\prime} \in[i, k]
\end{array}\right.
$$

because, by the subdivision property, after index $s$, either an interval $[i, k]$ is visited first, then an index $t^{\prime}$ of $[k+1, j]$, next the remaining indices of this last interval and finally index $t$, or the opposite, first an interval $[k+1, j]$, then an index $t^{\prime}$ in $[i, k]$, next the rest of the indices in that interval and finally index $t$.

The algorithm computes the $O\left(n^{4}\right)$ values $C(i, j, s, t)$ in increasing order of the size of the intervals $[i, j]$. Since every $C(i, j, s, t)$ requires $O\left(n^{2}\right)$ time, the overall complexity is $O\left(n^{6}\right)$ time and $O\left(n^{4}\right)$ space. The length of the shortest g-pyramidal cycle is

$$
\min _{k}(C(2, n, k, 1)+d(1, k)), k \in[2, n]
$$

and the shortest cycle is obtained in the standard way of dynamic programming.

Therefore, we have proved the following theorem.

Theorem 2. Given a distance matrix D, the shortest g-pyramidal cycle (or shortest twisted sequence) can be computed in $O\left(n^{6}\right)$ time and $O\left(n^{4}\right)$ space. 
As a consequence of this theorem, the BTSP for a complete bipartite graph $G$ satisfying the quadrangle property can be solved in $O\left(n^{6}\right)$ time. If $d(i, j)$ is the distance between vertices $i$ and $j$ of $G$, we only need to apply the previous algorithm with a distance matrix $D^{\prime}$ defined by $d^{\prime}(i, j)=d(i, j)$ if $i$ and $j$ have different colors and $d^{\prime}(i, j)=\infty$ if they have the same color. Therefore, we have the following theorem.

Theorem 3. Let $G=(R \cup B, E)$ be a complete bipartite graph such that $R \cup B$ is a partition of $V=\{1,2, \ldots, 2 n\}$, with $|R|=|B|=n$. Assume that $G$ satisfies the quadrangle property with respect to the cyclic order $(1,2, \ldots, 2 n)$. Then, a shortest Hamiltonian alternating cycle in $G$ can be computed in $O\left(n^{6}\right)$ time.

\section{Some particular cases satisfying the quadrangle property}

This section describes some particular cases of the BTSP satisfying the quadrangle property.

\subsection{The BTSP for Kalmanson matrices}

A $2 n \times 2 n$ symmetric distance matrix $D$ is a Kalmanson matrix if

$$
d(i, j)+d(k, l) \leq d(i, k)+d(j, l) \quad \text { and } \quad d(i, l)+d(j, k) \leq d(i, k)+d(j, l)
$$

for all $i, j, k, l$ with $1 \leq i<j<k<l \leq 2 n$. Kalmanson matrices are popular in the literature because the special structure of these matrices allows one to polynomially solve particular cases of certain problems. In Operations Research, the prize-collecting TSP [14] or the quadratic assignment problem [17] are some examples. In particular, if the distance matrix for the TSP is a Kalmanson matrix, then a shortest tour is the identity permutation [25]. Kalmanson matrices belong to a larger family of well-structured matrices defined by the so-called four-point inequalities. We refer the reader to [16] for a complete description of this family and the computational complexity of the TSP when the distance matrix belongs to the family.

The inequalities defining a Kalmanson matrix are quadrangle inequalities. Thus, assuming that $(1,2, \ldots, 2 n)$ is a cyclic order, it is clear that a complete graph satisfies the quadrangle property if and only if its underlying distance matrix is a Kalmanson matrix.

Let $G=(V, E)$ be a complete graph on the set of vertices $V=\{1,2, \ldots, 2 n\}$ and let $V=R \cup B$ be a partition of the vertices of $G$ into red and blue vertices, with $|R|=|B|=n$. Regardless of the partition of $V$ into two sets $R$ and $B$ of equal size, if the complete graph $G$ satisfies the quadrangle property with respect to the cyclic order $(1,2, \ldots 2 n)$, then the corresponding complete bipartite graph $G^{\prime}=\left(R \cup B, E^{\prime}\right)$ also satisfies it. As looking for the shortest Hamiltonian alternating cycle in $G$ is equivalent to looking for the shortest Hamiltonian alternating cycle in $G^{\prime}$, we have the following result by Theorem 3 .

Corollary 1. Let $G=(V, E)$ be a complete graph satisfying the quadrangle property with respect to the cyclic order $(1,2, \ldots, 2 n)$ (or equivalently, whose underlying distance matrix is a Kalmanson matrix). Let $V=R \cup B$ be a partition of the vertices of $V$ into two color classes $R$ and $B$ such that $|R|=|B|=n$. Then, a shortest Hamiltonian alternating cycle in $G$ can be computed in $O\left(n^{6}\right)$ time. 
The following lemma shows an additional property for an optimum Hamiltonian alternating cycle $C$ in a complete graph satisfying the quadrangle property: $C$ cannot contain three edges crossing each other. This result is applied in the next section to give a better algorithm when solving the BTSP for a circular graph.

A similar result is proved in [13] when computing a Hamiltonian alternating path $P$, minimizing the number of crossings in a bicolored convex point set in the plane: $P$ cannot contain three edges crossing each other. Using Observation 1, the proof given in [13] can be carried over to prove Lemma 4. For the sake of completeness, we include a proof which follows the lines of the proof appearing in [13] with some minor changes.

Lemma 4. Let $G=(V, E)$ be a complete graph satisfying the quadrangle property with respect to the cyclic order $(1,2, \ldots, 2 n)$. Let $V=R \cup B$ be a partition of the vertices of $V$ into two color classes $R$ and $B$ such that $|R|=|B|=n$. Then, no optimum Hamiltonian alternating cycle $C$ in $G$ can contain three edges $\left(i_{1}, j_{1}\right),\left(i_{2}, j_{2}\right)$ and $\left(i_{3}, j_{3}\right)$ crossing each other.

Proof. The proof of the lemma is again by contradiction: If there are three edges crossing each other, then one can build a new cycle shorter than $C$. By Observation 1, we can suppose that the vertices of $V$ are points ordered clockwise on a unit circle, so crossing edges are crossing segments.

Assume that $C$ contains three edges $\left(i_{1}, j_{1}\right),\left(i_{2}, j_{2}\right)$ and $\left(i_{3}, j_{3}\right)$ crossing each other and that $C=\left(i_{1}, j_{1}\right), P_{j_{1}, i_{2}},\left(i_{2}, j_{2}\right), P_{j_{2}, i_{3}},\left(i_{3}, j_{3}\right), P_{j_{3}, i_{1}}$, where $P_{i, j}$ is the path in $C$ connecting $i$ to $j$. By Lemma $2, i_{1}, i_{2}$ and $i_{3}$ must have the same color, say red. Thus, $j_{1}, j_{2}$ and $j_{3}$ are blue. There are 8 possible cyclic orders of placing these six colored points $i_{1}, j_{1}, i_{2}, j_{2}, i_{3}, j_{3}$ such that edges $\left(i_{1}, j_{1}\right),\left(i_{2}, j_{2}\right),\left(i_{3}, j_{3}\right)$ cross each other. By symmetry, they can be reduced to study the following 4 clockwise orders: (1) $i_{1}, i_{3}, i_{2}, j_{1}, j_{3}, j_{2}$, (2) $i_{1}, i_{2}, i_{3}, j_{1}, j_{2}, j_{3}$, (3) $i_{1}, j_{3}, i_{2}, j_{1}, i_{3}, j_{2}$ and (4) $i_{1}, i_{2}, j_{3}, j_{1}, j_{2}, i_{3}$. See Figure 4 . In all cases, by applying the quadrangle inequality two or three times, we can obtain a Hamiltonian alternating cycle shorter than $C$, which is a contradiction.

In case (1) (see the top left part of Figure 4 ), we first replace edges $\left(i_{2}, j_{2}\right)$ and $\left(i_{3}, j_{3}\right)$ by $\left(i_{2}, j_{3}\right)$ and $\left(i_{3}, j_{2}\right)$. Then, we replace $\left(i_{1}, j_{1}\right)$ and $\left(i_{3}, j_{2}\right)$ by $\left(i_{1}, j_{2}\right)$ and $\left(i_{3}, j_{1}\right)$ to obtain a new cycle $C^{\prime}=\left(i_{1}, j_{2}\right), P_{j_{2}, i_{3}}\left(i_{3}, j_{1}\right), P_{j_{1}, i_{2}},\left(i_{2}, j_{3}\right), P_{j_{3}, i_{1}}$. By Lemma $1, C^{\prime}$ is shorter than $C$ or has fewer crossings. In the figure, the dotted segments are the original edges $\left(i_{1}, j_{1}\right),\left(i_{2}, j_{2}\right)$ and $\left(i_{3}, j_{3}\right)$, the dashed segment represents the intermediate edge appearing after the first application of the quadrangle inequality, and the thick segments are the final edges belonging to the new cycle. Paths $P_{j_{1}, i_{2}}, P_{j_{2}, i_{3}}$ and $P_{j_{3}, i_{1}}$ are illustrated as curves. Further, numbers 1 and 2 represent the intersection point of the segments to which we apply the quadrangle inequality and the order in which we do it.

The constructions for the rest of the cases are summarized in Figure 4. In all cases, the meaning of the dotted segments, dashed segments and numbers is the same as explained previously in case (1). 

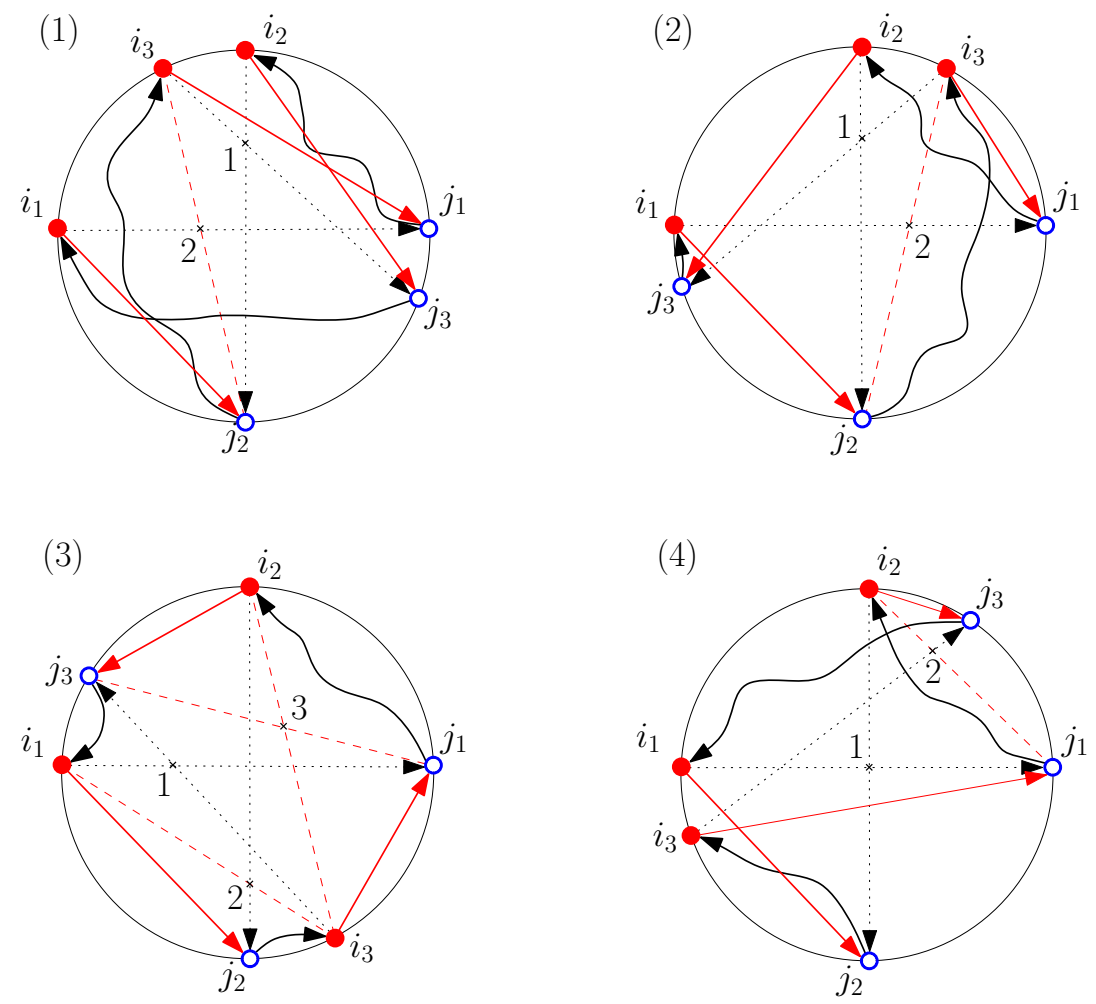

Figure 4: Constructing a new cycle with less length than $C$ when three edges cross: (1) cyclic order $i_{1}, i_{3}, i_{2}, j_{1}, j_{3}, j_{2}$, (2) cyclic order $i_{1}, i_{2}, i_{3}, j_{1}, j_{2}, j_{3}$, (3) cyclic order $i_{1}, j_{3}, i_{2}, j_{1}, i_{3}, j_{2}$ and (4) cyclic order $i_{1}, i_{2}, j_{3}, j_{1}, j_{2}, i_{3}$.

\subsection{The BTSP for a set of points in convex position}

Let $S$ be a set of $2 n$ points in the plane in convex position. Aligned points are allowed, so the points of $S$ are vertices of the convex hull of $S$ or are on the boundary of the convex hull of $S$. Assuming that the distance between two arbitrary points is the Euclidean distance, given a partition of $S=R \cup B$ into a set $R$ of $n$ red points and a set $B$ of $n$ blue points, we look for the shortest Hamiltonian alternating cycle visiting the points of $S$. Figure 1 shows the shortest Hamiltonian alternating cycles for those sets of points in convex position.

Suppose that $\left(p_{1}, p_{2}, \ldots, p_{2 n}\right)$ is the clockwise order of the points of $S$. Given four points $p_{i_{1}}<p_{i_{2}}<p_{i_{3}}<p_{i_{4}}$, clearly $d\left(p_{i_{1}}, p_{i_{4}}\right)+d\left(p_{i_{2}}, p_{i_{3}}\right) \leq d\left(p_{i_{1}}, p_{i_{3}}\right)+d\left(p_{i_{2}}, p_{i_{4}}\right)$ because the Euclidean distance satisfies the quadrangle inequality. Thus, we have the following corollary.

Corollary 2. Let $S=R \cup B$ be a bicolored convex point set in the plane such that $|R|=$ $|B|=n$. Then, a shortest Hamiltonian alternating cycle visiting the points of $S$ can be computed in $O\left(n^{6}\right)$ time. 


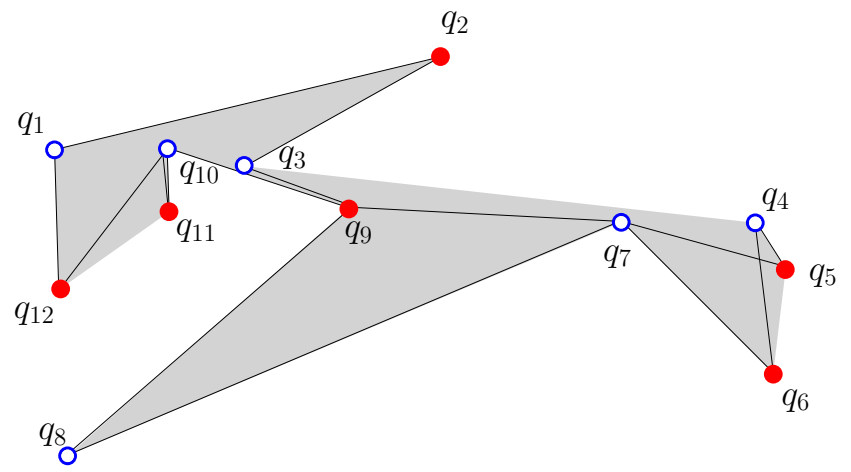

Figure 5: The shortest Hamiltonian alternating cycle $q_{1}, q_{2}, q_{3}, q_{9}, q_{7}, q_{5}, q_{4}, q_{6}, q_{8}, q_{11}, q_{10}, q_{12}$ for the simple polygon $q_{1}, q_{2}, q_{3}, q_{4}, q_{5}, q_{6}, q_{7}, q_{8}, q_{9}, q_{10}, q_{11}, q_{12}$.

\subsection{The BTSP for a simple polygon}

Let $S$ be a set of points in the plane (not necessarily in general position) and let $Q$ be a simple polygon whose vertices are the points of $S$. In a simple polygon $Q$, the distance between any two vertices of $Q$ is the geodesic distance, that is, the (Euclidean) length of the shortest path inside $Q$ between the two vertices. This path is called the geodesic path or edge between the two vertices. Given a partition $S=R \cup B$ of the vertices of $Q$ into red and blue vertices, with $|R|=|B|$, in the BTSP for $Q$ we look for the shortest alternating cycle $C$ inside $Q$ visiting its vertices. See Figure 5 . Note that, in $C$, a red vertex $p$ is visited after a blue vertex $q$, but, since the path from $q$ to $p$ is a geodesic path, it can contain some red and blue points not necessarily visited in an alternating way. For example, in Figure 5, the geodesic path from $q_{6}$ to $q_{8}$, the path $q_{6}, q_{7}, q_{8}$, passes through consecutive blue points.

Given four vertices $q_{1}, q_{2}, q_{3}, q_{4}$, ordered clockwise on $Q$, it is well-known by the Jordan curve theorem that the geodesic edges $q_{1} q_{3}$ and $q_{2} q_{4}$ must necessarily cross and can even share some segments, but geodesic edges $q_{1} q_{2}$ and $q_{3} q_{4}$ (or $q_{1} q_{4}$ and $q_{2} q_{3}$ ) can share some segments but they never properly cross. As a consequence, it is also well-known that the geodesic distance satisfies the quadrangle inequality, that is, if $d\left(q_{i}, q_{j}\right)$ is the geodesic distance between vertices $q_{i}$ and $q_{j}$, then, $d\left(q_{1}, q_{4}\right)+d\left(q_{2}, q_{3}\right) \leq d\left(q_{1}, q_{3}\right)+d\left(q_{2}, q_{4}\right)$. This last result follows from the fact that the geodesic edges $q_{1} q_{3}$ and $q_{2} q_{4}$ cross at a (first) point $p$. Then, one can define a path $c_{1}$ from $q_{1}$ to $q_{4}$, consisting of the part of $q_{1} q_{3}$ from $q_{1}$ to $p$ and the part of $q_{2} q_{4}$ from $p$ to $q_{4}$, and a path $c_{2}$ from $q_{2}$ to $q_{3}$, consisting of the part of $q_{2} q_{4}$ from $q_{2}$ to $p$ and the part of $q_{1} q_{3}$ from $p$ to $q_{3}$. Obviously, the sum of the lengths of $c_{1}$ and $c_{2}$ coincides with $d\left(q_{1}, q_{3}\right)+d\left(q_{2}, q_{4}\right)$, so $d\left(q_{1}, q_{4}\right)+d\left(q_{2}, q_{3}\right) \leq d\left(q_{1}, q_{3}\right)+d\left(q_{2}, q_{4}\right)$ by the minimality of the geodesic edges $q_{1} q_{4}$ and $q_{2} q_{3}$.

Therefore, since the complete graph $G=(S, E)$ satisfies the quadrangle property with the geodesic distance and the clockwise order of the vertices of $Q$, the following result holds.

Corollary 3. Let $S=R \cup B$ be a bicolored point set such that $|R|=|B|=n$ and the points of $S$ are the vertices of a simple polygon $Q$. Then, a shortest Hamiltonian alternating cycle inside $Q$ can be computed in $O\left(n^{6}\right)$ time. 
Given an $n$-vertex simple polygon $Q$, we remark that the geodesic distance between two arbitrary vertices can be computed in $O(\log n)$ time, after a linear preprocessing (see for example [22] for details). Thus, all the geodesic distances can be precomputed in $O\left(n^{2} \log n\right)$ time. We also remark that a similar result is obtained when $n$ red points and $n$ blue points are placed on the boundary of a $k$-vertex simple polygon $Q$ and one looks for the shortest Hamiltonian alternating cycle $C$ inside $Q$ visiting the $2 n$ points: $C$ can be computed in $O\left(n^{6}+n^{2} \log k+k\right)$ time.

\subsection{The BTSP for a circular graph}

Let $G=(V, E)$ be an undirected graph, with $V=\{1,2, \ldots, n\}$ as its set of $n$ vertices. Assuming that $n$ is followed by 1, $G$ is called a circular graph if $E$ consists of the $n$ edges $(i, i+1)=(i+1, i)$, for $i=1, \ldots, n$. For every edge $(i, i+1)$, a non-negative real number $l_{i}$, the length of the edge, is given. As in the case of the simple polygon, we define the geodesic edge $(i, j)$ between vertex $i$ and vertex $j$ (or shortest path from $i$ to $j$ in $G$ ), as path $i, i+1, \ldots, j$ if $\sum_{k=i}^{k=j-1} l_{k} \leq \sum_{k=j}^{k=i-1} l_{k}$, and path $j, j+1, \ldots, i$ otherwise. The geodesic distance between $i$ and $j$ is the sum of lengths of the edges in $(i, j)$. Note that geodesic edges $(i, j)$ and $(j, i)$ coincide, so the geodesic distances between $i$ and $j$ and between $j$ and $i$ also coincide. For a partition of the set of vertices $V=R \cup B$ (the red and the blue vertices), with $|R|=|B|=n$, in the BTSP for a circular graph we look for the shortest Hamiltonian alternating cycle.

All the geodesic distances can be precomputed easily in $O\left(n^{2}\right)$ time and it is quite clear that the geodesic distance for a circular graph also satisfies the quadrangle inequality, that is, $d\left(i_{1}, i_{4}\right)+d\left(i_{2}, i_{3}\right) \leq d\left(i_{1}, i_{3}\right)+d\left(i_{2}, i_{4}\right)$ for any four vertices $i_{1}, i_{2}, i_{3}, i_{4}$ such that $i_{1}<i_{2}<i_{3}<i_{4}$ cyclically. Therefore, the complete graph $K$ consisting of the vertices of $G$ satisfies the quadrangle property with respect to the cyclic order $(1,2, \ldots, n)$ on its vertices and the distance between two vertices of $K$ defined as the geodesic distance between these two vertices in $G$. By Lemmas 2 and 4, and Theorems 1 and 3, the following result holds.

Corollary 4. Let $G=(V, E)$ be a bicolored circular graph such that $V=R \cup B$ and $|R|=|B|=n$. If $C$ is an optimum Hamiltonian alternating cycle in $G$ then:

i) $C$ cannot contain a five-point star.

ii) If the directed geodesic edges $(i, j)$ and $\left(i^{\prime}, j^{\prime}\right)$ of $C$ cross, then $i, i^{\prime}$ have the same color.

iii) $C$ cannot contain three geodesic edges crossing each other.

Moreover, a shortest Hamiltonian alternating cycle in $G$ can be computed in $O\left(n^{6}\right)$ time.

\section{An $O\left(n^{2}\right)$ algorithm for the BTSP in a circular graph}

In this section we provide an $O\left(n^{2}\right)$ algorithm to solve the BTSP for a circular graph. The algorithm is based on proving that, for any optimum cycle $C$, there is an edge of the circular graph visited at most once in $C$. Then, the linear algorithm for the BTSP on a line graph [31] can be used as a subroutine. The fact that an edge of the circular graph is 
visited at most once in optimum cycles is a consequence of the fact that, by Corollary 4 , such cycles do not contain either a five-point star or three edges crossing each other.

The next lemma gives a specific way in which the different indices are visited in a g-pyramidal permutation.

Lemma 5. Let $\pi=\left(i_{1}, i_{2}, \ldots, i_{n}\right)$ be a g-pyramidal permutation and let $A$ and $B$ be the intervals $\left(i_{1}, i_{2}\right)$ and $\left(i_{2}, i_{1}\right)$, respectively. Suppose that $i_{1}$ and $i_{2}$ are not consecutive indices and that, after $i_{2}$, an index in $A$ is visited in $\pi$. Then, $A$ and $B$ can be divided into $k$ disjoint intervals $A_{1}=\left[j_{1}, i_{2}\right), A_{2}=\left[j_{2}, j_{1}\right), \ldots, A_{k}=\left(i_{1}, j_{k-1}\right)$ and $B_{1}=\left(i_{2}, j_{1}^{\prime}\right], B_{2}=$ $\left(j_{1}^{\prime}, j_{2}^{\prime}\right], \ldots, B_{k}=\left(j_{k-1}, i_{1}\right)$, with $i_{1}<j_{k-1}<j_{k-2}<\ldots<j_{1}<i_{2}$ and $i_{2}<j_{1}^{\prime}<j_{2}^{\prime}<\ldots<$ $j_{k-1}^{\prime}<i_{1}$, such that $\pi$ visits the intervals in the order $i_{1}, i_{2}, A_{1}, B_{1}, A_{2}, B_{2}, \ldots, A_{k}, B_{k}$. Moreover, if the index visited in $\pi$ before $i_{1}$ belongs to $A$, then $B_{k}=\emptyset$.

Proof. After $i_{2}$, the indices in $A$ and $B$ are visited alternately in $\pi$ before reaching $i_{1}$ : after $i_{2}$, first a set $A_{1} \subseteq A$, followed by a set $B_{1} \subseteq B$, then a set $A_{2} \subset A$ and so on until $i_{1}$ is reached either from an index in $A_{k}$ (and then $B_{k}=\emptyset$ ) or from an index in $B_{k}$. Suppose that $B_{k} \neq \emptyset$ (if $B_{k}=\emptyset$ the reasoning is analogous). Let $I_{i}=A_{1} \cup A_{2} \cup \ldots \cup A_{i}$ and let $s_{i}$ be the index in $I_{i}$ such that the interval $\left(i_{1}, s_{i}\right)$ does not contain any index of $I_{i}$. Let us prove that, for $i=1, \ldots, k$, the set $I_{i}$ consists of all the indices in the interval $\left[s_{i}, i_{2}\right)$. Let $b_{i}$ be the first index of $B_{i}$ visited in $\pi$ from $i_{2}$. If an index $i_{3}$ in the interval $\left[s_{i}, i_{2}\right)$ is not visited in $\pi$ between $i_{2}$ and $b_{i}$, then the five indices $i_{1}<s_{i}<i_{3}<i_{2}<b_{i}$ are visited in $\pi$ in the order $\left(i_{1}, i_{2}, \ldots, s_{i}, \ldots, b_{i}, \ldots, i_{3}, \ldots\right)$, contradicting that $\pi$ is g-pyramidal. Therefore, $A_{1}=\left[j_{1}, i_{2}\right), A_{2}=\left[j_{2}, j_{1}\right), \ldots, A_{k}=\left(i_{1}, j_{k-1}\right)$, with $i_{1}<j_{k-1}<j_{k-2}<\ldots<j_{1}<i_{2}$. The same reasoning applies to show that $B_{1}=\left(i_{2}, j_{1}^{\prime}\right], B_{2}=\left(j_{1}^{\prime}, j_{2}^{\prime}\right], \ldots, B_{k}=\left(j_{k-1}, i_{1}\right)$, with $i_{2}<j_{1}^{\prime}<j_{2}^{\prime}<\ldots<j_{k-1}^{\prime}<i_{1}$.

Using this result, Lemma 6 shows that, for an optimum Hamiltonian alternating cycle $C$, there is an edge of the circular graph which is visited at most once in $C$.

Lemma 6. Let $G=(V, E)$ be a bicolored circular graph such that $V=R \cup B$ and $|R|=$ $|B|=n$. Let $C$ be an optimum Hamiltonian alternating cycle in $G$. Then, there is an edge of $G$ visited at most once in $C$.

Proof. Recall that a geodesic edge is the shortest path in $G$ between the endpoints of the geodesic edge. Thus, if $l \leq l^{\prime}<m^{\prime} \leq m$ cyclically and $l^{\prime}$ and $m^{\prime}$ belong to the shortest path from $l$ to $m$, then the geodesic edge $\left(l^{\prime}, m^{\prime}\right)$ is contained in the geodesic edge $(l, m)$, that is, the shortest path from $l^{\prime}$ to $m^{\prime}$ is included in the shortest path from $l$ to $m$.

Suppose first that $C=(1,2, \ldots, n)$, that is, the vertices of $G$ are visited consecutively. If the geodesic edge $(i, i+1)$ is the path $i, i+1$, for all $i$, then every edge of $G$ is visited exactly once in $C$ and the theorem holds. On the other hand, if a geodesic edge $(i, i+1)$ is the path $i, i-1, \ldots, i+1$, then any other geodesic edge $(j, j+1)$ of $C$ must coincide with the path $j, j+1$. Hence, the edge $(i, i+1)$ is not used in $C$ and again the theorem holds.

Therefore, we may assume that $C=\left(i_{1}, i_{2}, \ldots\right)$, with $i_{1}$ and $i_{2}$ being non-adjacent vertices of $G$. In addition, without loss of generality, we can suppose that the geodesic 


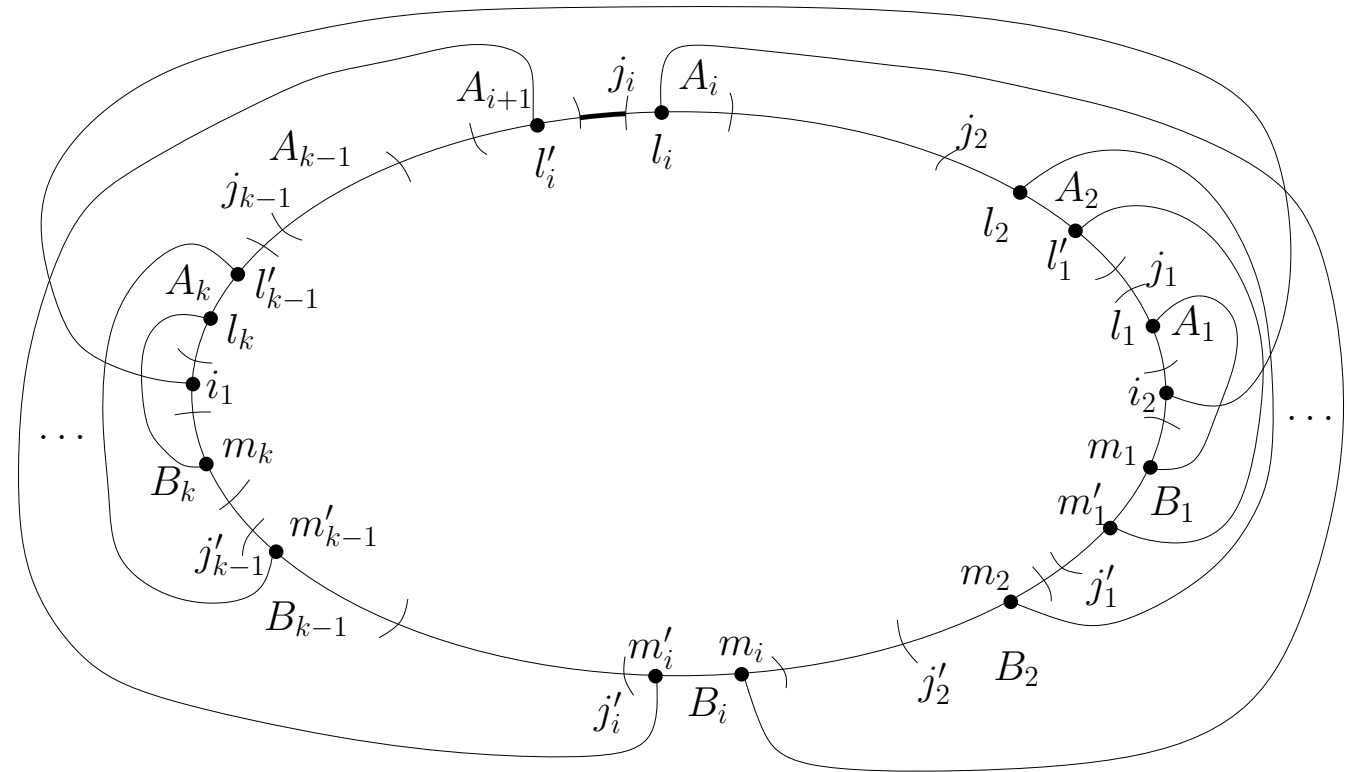

Figure 6 : Edge $\left(j_{i}-1, j_{i}\right)$ is visited exactly once in $C$ and by the geodesic edge $\left(i_{1}, i_{2}\right)$. The geodesic edges are illustrated as curves.

edge $\left(i_{1}, i_{2}\right)$ is the path $i_{1}, i_{1}+1, \ldots, i_{2}-1, i_{2}$ and that $\left(i_{1}, i_{2}\right)$ is maximal in the sense that the path $i_{1}, i_{1}+1, \ldots, i_{2}-1, i_{2}$ is not contained in any other geodesic edge of $C$.

Let $A$ and $B$ be the sets of vertices $\left\{i_{1}+1, i_{1}+2, \ldots, i_{2}-1\right\}$ and $\left\{i_{2}+1, i_{2}+2, \ldots, i_{1}-1\right\}$, respectively. By Corollary $4, C$ is g-pyramidal. Then, by Lemma 5 , the sets $A$ and $B$ can be decomposed into disjoint sets of consecutive vertices $A_{1}=\left\{j_{1}, \ldots, i_{2}-1\right\}, A_{2}=\left\{j_{2}, \ldots, j_{1}-\right.$ $1\}, \ldots, A_{k}=\left\{i_{1}+1, \ldots, j_{k-1}-1\right\}$ and $B_{1}=\left\{i_{2}+1, \ldots, j_{1}^{\prime}\right\}, B_{2}=\left\{j_{1}^{\prime}+1, \ldots, j_{2}^{\prime}\right\}, \ldots, B_{k}=$ $\left\{j_{k-1}^{\prime}+1, \ldots, i_{1}-1\right\}$, with $i_{1}<j_{k-1}<j_{k-2}<\ldots<j_{1}<i_{2}$ and $i_{2}<j_{1}^{\prime}<j_{2}^{\prime}<\ldots<j_{k-1}^{\prime}<$ $i_{1}$, such that $C$ visits the sets in the order $i_{1}, i_{2}, A_{1}, B_{1}, A_{2}, B_{2}, \ldots, A_{k}, B_{k}$. See Figure 6 . We can suppose that $B_{k} \neq \emptyset$. If $B_{k}=\emptyset$ the reasoning is analogous.

Let $\left(l_{i}, m_{i}\right)$ be the geodesic edge connecting $A_{i}$ to $B_{i}$, for $i=1, \ldots, k$, and let $\left(m_{i}^{\prime}, l_{i}^{\prime}\right)$ be the geodesic edge connecting $B_{i}$ to $A_{i+1}$, for $i=1, \ldots, k-1$. By Corollary 4 , three geodesic edges cannot cross each other. Since $\left(l_{1}, m_{1}\right)$ and $\left(m_{1}^{\prime}, l_{1}^{\prime}\right)$ cross $\left(i_{1}, i_{2}\right)$, then $\left(l_{1}, m_{1}\right)$ and $\left(m_{1}^{\prime}, l_{1}^{\prime}\right)$ cannot cross, so the cyclic order of $i_{1}, i_{2}, l_{1}, m_{1}, m_{1}^{\prime}, l_{1}^{\prime}$ must be $i_{1}, l_{1}^{\prime}, l_{1}, i_{2}, m_{1}, m_{1}^{\prime}$. For the same reason, as $\left(m_{1}^{\prime}, l_{1}^{\prime}\right)$ and $\left(l_{2}, m_{2}\right)$ cannot cross, the cyclic order of $i_{1}, i_{2}, m_{1}^{\prime}, l_{1}^{\prime}, l_{2}, m_{2}$ must be $i_{1}, l_{2}, l_{1}^{\prime}, i_{2}, m_{1}^{\prime}, m_{2}$. Iterating this reasoning for the rest of the geodesic edges $\left(l_{i}, m_{i}\right)$ and $\left(m_{i}^{\prime}, l_{i}^{\prime}\right)$, necessarily the cyclic order of the endpoints of these geodesic edges is $i_{1}, l_{k}, l_{k-1}^{\prime}, l_{k-1}, \ldots, l_{1}^{\prime}, l_{1}, i_{2}, m_{1}, m_{1}^{\prime}, m_{2}, m_{2}^{\prime}, \ldots, m_{k}$. Now, consider the following ordered sequence of geodesic edges: $S E=\left(l_{k}, m_{k}\right),\left(m_{k-1}^{\prime}, l_{k-1}^{\prime}\right),\left(l_{k-1}, m_{k-1}\right), \ldots,\left(m_{1}^{\prime}, l_{1}^{\prime}\right),\left(l_{1}, m_{1}\right)$. To show that an edge of $G$ is visited at most once in $C$, we study several cases depending on the way in which $i_{1}$ and $i_{2}$ belong to the geodesic edges of $S E$.

Suppose first that there exist two consecutive geodesic edges in $S E,\left(m_{i}^{\prime}, l_{i}^{\prime}\right),\left(l_{i}, m_{i}\right)$, such that $i_{1}$ belongs to $\left(m_{i}^{\prime}, l_{i}^{\prime}\right)$ and $i_{2}$ belongs to $\left(l_{i}, m_{i}\right)$, that is, $\left(m_{i}^{\prime}, l_{i}^{\prime}\right)$ is the path $m_{i}^{\prime}, m_{i}^{\prime}+1, \ldots, l_{i}^{\prime}-1, l_{i}^{\prime}$ and $\left(l_{i}, m_{i}\right)$ is the path $l_{i}, l_{i}+1, \ldots, m_{i}-1, m_{i}$. Let us see that 
edge $e=\left(j_{i}-1, j_{i}\right)$ is visited at most once in $C$. As the cyclic order of the endpoints of the geodesic edges of $S E$ is $i_{1}, l_{k}, l_{k-1}^{\prime}, l_{k-1}, \ldots, l_{1}^{\prime}, l_{1}, i_{2}, m_{1}, m_{1}^{\prime}, m_{2}, m_{2}^{\prime}, \ldots, m_{k}$, then any geodesic edge appearing in $S E$ before $\left(m_{i}^{\prime}, l_{i}^{\prime}\right)$ is contained in $\left(m_{i}^{\prime}, l_{i}^{\prime}\right)$ and any geodesic edge appearing in $S E$ after $\left(l_{i}, m_{i}\right)$ is contained in $\left(l_{i}, m_{i}\right)$. As a consequence, edge $e$ does not belong to any geodesic edge of $S E$. By assumption, the geodesic edge $\left(i_{1}, i_{2}\right)$ is the path $\left(i_{1}, i_{1}+1, \ldots, i_{2}-1, i_{2}\right)$, so $e$ belongs to the geodesic edge $\left(i_{1}, i_{2}\right)$. But then, a geodesic edge $e^{\prime}$ of $C$ connecting two vertices in $B$ cannot contain $e$ because, otherwise, the geodesic edge $\left(i_{1}, i_{2}\right)$ would be contained in $e^{\prime}$, contradicting the maximality of $\left(i_{1}, i_{2}\right)$. Moreover, a geodesic edge $e^{\prime}$ of $C$ connecting two vertices in $A_{j}$, for all $j$, neither can contain $e$ because, otherwise, $i_{1}, i_{1}+1, \ldots, i_{2}-1, i_{2}$ would not be the shortest path between $i_{1}$ and $i_{2}$. Therefore, edge $\left(j_{i}-1, j_{i}\right)$ is visited exactly once in $C$ and by the geodesic edge $\left(i_{1}, i_{2}\right)$.

An identical analysis can be done if there are two consecutive geodesic edges in $S E$, $\left(l_{i}, m_{i}\right)$ and $\left(m_{i-1}^{\prime}, l_{i-1}^{\prime}\right)$, such that $i_{1}$ belongs to $\left(l_{i}, m_{i}\right)$ and $i_{2}$ to $\left(m_{i-1}^{\prime}, l_{i-1}^{\prime}\right)$ : edge $\left(j_{i-1}^{\prime}, j_{i-1}^{\prime}+\right.$ $1)$ is visited at most once. Finally, edge $\left(i_{2}, i_{2}+1\right)$ is visited at most once if $i_{1}$ belongs to all the geodesic edges of $S E$ and edge $\left(i_{1}-1, i_{1}\right)$ is visited at most once if $i_{2}$ belongs to all the geodesic edges of $S E$.

Using this lemma, the $O\left(n^{2}\right)$ algorithm to solve the BTSP for a circular graph is as follows. In [31], the authors give a linear algorithm to solve the BTSP on a line graph $L G$. Their algorithm is based on giving a lower bound on the number of times that any Hamiltonian alternating cycle must visit every edge of the line graph, and then on building a cycle reaching that lower bound. If $(1,2),(2,3), \ldots,(n-1, n)$ is the set of edges of the line graph $L G$, the same approach works to find in linear time the shortest Hamiltonian alternating path from vertex 1 to vertex $n$.

According to Lemma 6 , there is an edge $e=(i, i+1)$ of $G$ visited at most once in an optimum Hamiltonian alternating cycle $C$. If $e$ is not visited in $C$, then, by removing $e$, the BTSP for a circular graph is equivalent to the BTSP for a line graph, which can be solved in linear time.

Assume then that $e$ is visited once in $C$ and suppose without loss of generality that the geodesic edge $\left(j_{1}, j_{2}\right)$ of $C$ is the only one containing edge $e$, and that $j_{1}$ is blue and $j_{2}$ is red. From $G$, we can define a line graph $L G$ as follows: We add two new vertices $i^{\prime}$ and $i^{\prime}+1$ to $G$, color vertex $i^{\prime}$ in red and vertex $i^{\prime}+1$ in blue, remove the edge $(i, i+1)$ with length $l_{i}$, and add the edges $\left(i, i^{\prime}\right)$ and $\left(i^{\prime}+1, i+1\right)$, with length 0 each.

In linear time, we can find the shortest Hamiltonian alternating path $P_{1}=i^{\prime}, i_{1}, \ldots, i_{n}, i^{\prime}+$ 1 in $L G$, from vertex $i^{\prime}$ to vertex $i^{\prime}+1$. From $P_{1}$, by removing the paths $i^{\prime}, i, \ldots, i_{1}$ from $i^{\prime}$ to $i_{1}$ and $i_{n}, i_{n}-1, \ldots, i+1, i^{\prime}+1$ from $i_{n}$ to $i^{\prime}+1$, and by adding the path $i_{1}, i_{1}+1, \ldots, i, i+1, \ldots, i_{n}$ in $G$ from $i_{1}$ to $i_{n}$, we obtain a Hamiltonian alternating cycle $C_{1}=\left(i_{1}, \ldots, i_{n}\right)$ in $G$. Note that $l_{i}$ is the difference between the lengths of $C_{1}$ and $P_{1}$.

Now, observe that by removing the geodesic edge $\left(j_{1}, j_{2}\right)$ from $C$ and by adding the paths $j_{1}, j_{1}+1, \ldots, i, i^{\prime}$ from $j_{1}$ to $i^{\prime}$ and $j_{2}, j_{2}-1, \ldots, i+1, i^{\prime}+1$ from $j_{2}$ to $i^{\prime}+1$, we obtain a Hamiltonian alternating path $P$ in $L G$ from $i^{\prime}$ to $i^{\prime}+1$. Again, the difference between the lengths of $C$ and $P$ is $l_{i}$, so necessarily the length of $C_{1}$ must coincide with the length of $C$. 
Therefore, to solve the BTSP for a circular graph knowing the edge $e=(i, i+1)$ which is visited at most once in $C$, we only need to solve three instances of the BTSP for a line graph. If $e$ is not used in $C$, we solve the BTSP for a line graph after removing edge $e$. If $e$ is visited exactly once in $C$, we solve two instances of the BTSP for a line graph, coloring $i^{\prime}$ in red and $i^{\prime}+1$ in blue or the opposite, because we do not know a priori the colors of the endpoints of the geodesic edge of $C$ using $e$. As there are $n$ possible choices for $e$, the following theorem holds.

Theorem 4. The BTSP for a circular graph can be solved in $O\left(n^{2}\right)$ time.

\section{Concluding remarks}

In this paper, we have shown that, if the quadrangle property holds in the BTSP for a complete bipartite graph, any optimum cycle must be g-pyramidal. We have also shown how to compute in $O\left(n^{6}\right)$ time the shortest g-pyramidal permutation, given a distance matrix, hence this special case of the BTSP can be solved in $O\left(n^{6}\right)$ time. Moreover, using some additional properties, it is possible to give an $O\left(n^{2}\right)$ algorithm to solve the BTSP in a circular graph.

There are several interesting questions for future work, related to this research. A first question is whether an algorithm to find the shortest g-pyramidal cycle whose time complexity is less than $O\left(n^{6}\right)$ exists. If this is the case, any problem whose solution is g-pyramidal could be solved with such complexity. A second question is whether Lemma 4 (or any other additional property) allows one to design a better algorithm to solve the BTSP for a complete graph satisfying the quadrangle property, as we did for the case of circular graphs. A third question is to find new problems admitting optimal solutions which are g-pyramidal, so they could be solved in $O\left(n^{6}\right)$ time. In particular, the complexity of the swapping problem and the $k$-DTSP for a circular graph are unknown, and perhaps the ideas given in this paper could be useful for throwing light on this question.

\section{Acknowledgements}

The authors express their sincere thanks to the anonymous referees for their insightful comments, which in particular helped us very much to improve the presentation of the results in this paper.

\section{References}

[1] S. Anily and J. Bramel, Approximation algorithms for the capacitated traveling salesman probem with pickups and deliveries. Naval Res Logistics 56 (1999), 654-670.

[2] S. Anily, M. Gendreau and G. Laporte, The swapping problem on a line. SIAM J. Comput. 29 (1999), 327-335.

[3] S. Anily, M. Gendreau and G. Laporte, The pre-empitve swapping problem on a tree. Networks 58 (2011), 83-94. 
[4] S. Anily and R. Hassin, The swapping problem. Networks 22 (1992), 11-18.

[5] S. Anily and A. Pfeffer, The uncapacitated swapping problem on a line and on a circle. Discrete Applied Mathematics 161 (2013), 454-465.

[6] F. Aurenhammer, On-line sorting of twisted sequences in linear time. BIT, 28 (1988), 194-204

[7] A. Baltz and A. Srivastav, Aproximation algorithms for the Euclidean bipartite TSP. Operations Research Letters 33 (2005), 403-410.

[8] G. Berbeglia, J.F. Cordeau, I. Gribkovskaia and G. Laporte, Static pickup and delivery problems: a classification scheme and survey. TOP 15 (2007), 1-31.

[9] B. Bhattacharya and Y. Hu, k-delivery traveling salesman problem on tree networks. In: IARCS Annual Conference on Foundations of Software Technology and Theoretical Computer Science (FSTTCS 2012), 2012, 325-336. DOI: 10.4230/LIPIcs.FSTTCS.2012.325.

[10] R.E. Burkard, V.G. Deineko, R. van Dal, J.A.A. van der Veen and G.J. Woeginger, Well-solvable special cases of the TSP: A survey. SIAM Rev. 40 (1998), 496-546.

[11] P. Chalasani, R. Motwani, Approximating capacitated routing and delivery problems. SIAM J. Comput. 28 (1999), 2133-2149.

[12] P. Chalasani, R. Motwani and A. Rao, Algorithms for robot grasp and delivery. In: Proceedings of the 2nd International Workshop on Algorithmic Foundations of Robotics, 1996, 347-362.

[13] M. Claverol, A. García, D. Garijo, C. Seara and J. Tejel, On Hamiltonian alternating cycles and paths. ArXiv:1603.06764

[14] S. Coene, C. Filippi, F.C.R. Spieksma and E. Stevanato, Balancing profits and costs on trees. Networks 61 (2013), 200-211.

[15] R.K. Congram, Polynomially Searchable Exponential Neighbourhoods for Sequencing Problems in Combinatorial Optimisation. PhD Thesis, University of Southampton, 2000. http://eprints.soton.ac.uk/50630/1.hasCoversheetVersion/00183297.pdf.

[16] V.G. Deineko, B. Klinz, A. Tiskin and G.J. Woeginger, Four-point condition for the TSP: The complete complexity classification. Discrete Optimization 14 (2014), 147159.

[17] V.G. Deineko and G.J. Woeginger, A solvable case of the quadratic assignement problem. Oper. Res. Lett. 22 (1998), 13-17. 
[18] V.G. Deineko and G.J. Woeginger, A study of exponential neighborhoods for the travelling salesman problem and for the quadratic assignment problem. Mathematical Programming 87 (2000), 519-542.

[19] V.G. Deineko and G.J. Woeginger, Another look at the shoelace TSP: The case of very old shoes. In: Fun with Algorithms (A. Ferro, F. Luccio and P. Widmayer, eds.), LNCS 8496 (2014), 125-136.

[20] A. Frank, B. Korte, E. Triesch and J. Vygen, On the bipartite traveling salesman problem. Technical Report No. 98866-OR. Research Institute for Discrete Mathematics, University of Bonn, Germany (1998).

[21] A. García and J. Tejel, The order of points on the second convex hull of a simple polygon. Discrete and Computational Geometry 14 (1995), 185-205.

[22] L. Guibas and J. Hershberger, Optimal shortest path queries in a simple polygon. J. Comput. Syst. Sci. 39 (1989), 126-152.

[23] G. Gutin abd A.P. Punnen, editors, The Traveling Salesman Problem and Its Variations. Springer, New York, 2007.

[24] J.H. Halton, The shoelace problem. The Mathematical Intelligencer 17 (1995), 36-41.

[25] K. Kalmanson, Edgeconvex circuits and the traveling salesman problem. Canad. J. Math. 27 (1975), 1000-1010.

[26] E.L. Lawler, J.K. Lenstra, A.H.G. Rinooy and D.B. Shmoys, editors, Traveling Salesman Problem: A Guided Tour of Combinatorial Optimization. Wiley, Chichester, 1985.

[27] M. Misiurewicz, Lacing irregular shoes. The Mathematical Intelligencer 18 (1996), $32-34$.

[28] A. Shurbevski, H. Nagamochi and Y. Karuno, Approximating the bipartite TSP and its biased generalization, in: WALCOM 2014 (S.P. Pal and K. Sadakane Eds.), Lecture Notes in Computer Science 8344 (2014), 56-67.

[29] A. Srivastav, H. Schroeter and C. Michel, Approximation algorithms for pick-andplace robots. Annals of Operations Research 107 (2001), 321-338.

[30] J. Tejel, Algoritmos polinomiales para algunos problemas de recorrido óptimo. PhD Thesis, University of Zaragoza, 1994 (in Spanish).

[31] F. Wang, A. Lim and Z. Xu, The one-commodity pickup and delivery travelling salesman problem on a path or a tree. Networks 48 (2006), 24-35. 